\title{
Artificial Intelligence and Computational Approaches for Epilepsy
}

\section{Review}

Journal of Epilepsy Research pISSN 2233-6249 / elSSN 2233-6257

Received June 8, 2020

Revised June 18, 2020

Accepted July 14, 2020

Corresponding author:

Hyang Woon Lee, MD, PhD

Departments of Neurology and Medical

Science, Ewha Womans University School of Medicine and Ewha Medical Research

Institute, 1071 Anyangcheon-ro,

Yangcheon-gu, Seoul 07985, Korea

Tel. $+82-2-2650-2673$

Fax. +82-2-2650-5958

E-mail; leeh@ewha.ac.kr

\author{
Sora An, PhD ${ }^{1,2}$, Chaewon Kang, BS ${ }^{1,3}$, Hyang Woon Lee, MD, PhD ${ }^{1,2,3}$ \\ Departments of ${ }^{1}$ Neurology, ${ }^{2}$ Medical Science, and ${ }^{3}$ Computational Medicine, Ewha Womans University School of \\ Medicine and Ewha Medical Research Institute, Seoul, Korea
}

\begin{abstract}
Studies on treatment of epilepsy have been actively conducted in multiple avenues, but there are limitations in improving its efficacy due to between-subject variability in which treatment outcomes vary from patient to patient. Accordingly, there is a growing interest in precision medicine that provides accurate diagnosis for seizure types and optimal treatment for an individual epilepsy patient. Among these approaches, computational studies making this feasible are rapidly progressing in particular and have been widely applied in epilepsy. These computational studies are being conducted in two main streams: 1) artificial intelligence-based studies implementing computational machines with specific functions, such as automatic diagnosis and prognosis prediction for an individual patient, using machine learning techniques based on large amounts of data obtained from multiple patients and 2) patient-specific modeling-based studies implementing biophysical in-silico platforms to understand pathological mechanisms and derive the optimal treatment for each patient by reproducing the brain network dynamics of the particular patient per se based on individual patient's data. These computational approaches are important as it can integrate multiple types of data acquired from patients and analysis results into a single platform. If these kinds of methods are efficiently operated, it would suggest a novel paradigm for precision medicine. (2020;10:8-17)
\end{abstract}

Key words: Epilepsy, Precision medicine, Seizures, Artificial intelligence, Patient-specific modeling

\section{Introduction}

Epilepsy is a chronic neurological disease that affects more than 50 million people worldwide. ${ }^{1}$ Epilepsy is defined as recurrence of unexpected seizures, which results in various ictal symptoms depending on the brain regions where the seizure is originated and propagated. ${ }^{2,3}$ It seriously degrades the quality of life not only of the individual patients, but of their family, which causes major socio-economic losses. ${ }^{4-6}$

For the treatment of epilepsy, medical therapy can be applied by priority, in which $60-70 \%$ of the patients have responded by single or multiple antiepileptic medications. ${ }^{7,8}$ For the drug-resistant patients, however, surgical intervention has been provided as an option, including resection that removes the focal area in the brain, which generates epileptic seizures, as well as disconnection that blocks the main neural pathways of seizure propagation. ${ }^{9-13}$ Although the epilepsy surgery has been accepted as an effective method to control the drug-resistant seizures, the postoperative outcomes have been largely variable depending on various clinical factors of the individual epilepsy patient, including etiology, diagnostic modalities and even decision makings by physicians or surgeons' point of view. ${ }^{9-13}$ The possibility of cognitive impairment after surgery caused by the removal or severance of specific brain areas is another important issue to consider. ${ }^{14-17}$

Recently, neuromodulation therapy using various brain stimulation modalities, including deep brain stimulation, vagal nerve stimulation, or external responsive neurostimulation, have been attempted to control intractable seizures. ${ }^{18-22}$ Those therapies modulate brain functions or pathological states at the entire brain network level, by stimulating the specific brain region to induce changes not only in the stimulated brain regions but also in distant areas that are connected to them through the anatomical or functional brain connectivity. While the neuromodulation therapies have been known as novel and promising treatment methods, demonstrating seizure reduction in 
about one-third to one-half of patients, ${ }^{18-22}$ there are still many obstacles for improving the treatment efficacy by finding the optimal stimulation sites or stimulation parameters, or understanding the mechanisms behind the treatment. ${ }^{23,24}$

Although studies for the epilepsy treatment have been actively conducted in multiple avenues, one of the most difficult challenges is the between-subject variability of the treatment outcomes. In fact, the treatment outcomes have been varied from patient to patient depending on the patient-specific intrinsic characteristics, including seizure types and semiologies, brain lesions, or comorbid neuropsychological dysfunctions, extrinsic factors such as the stage in which the treatment is first applied, and the treatment conditions. ${ }^{25-28}$ Therefore, personalized approaches that diagnose each patient's state accurately and choose an optimal treatment method to the individual patient are crucial.

With the advance of high-performance computing technologies and the development of numerous mathematical algorithms, computational studies for clinical application on epilepsy are being actively conducted to analyze large amounts of data, from which a proper solution can be derived for an individual patient (Fig. 1). These computational studies have significant impacts as it can provide automated and standardized protocols to support clinical decisions. This manuscript provides an overall introduction regarding recent computational studies on personalized medicine and discuss its future directions for diagnosis and treatment of epilepsy patients.

\section{Machine learning-based approaches}

Machine learning, an application of artificial intelligence (Al) technique, enables a machine to automatically learn something new by combining statistics and computer science and thus improve its performance through meaningful data, without explicit instruction. ${ }^{29}$ These learning tasks are executed in two main types: supervised versus unsupervised learnings..$^{29-33}$ Supervised learning is the approach that trains using labeled data, that is, data whose target outputs have already been known. It is mainly used for classification or regression purposes and its algorithm includes k-nearest neighbor (k-NN), linear/logistic regression, naïve Bayes, random forest, and support vector machine (SVM). ${ }^{29-33}$ On the other hand, unsupervised learning is the approach that trains using unlabeled data. It is mainly used for clustering or association analysis purposes and its algorithm includes k-means, k-medoids, fuzzy C-means, Gaussian mixture, hidden Markov model. ${ }^{29-33}$ Artificial neural network (ANN) is another machine learning algorithm that performs the learning task by mimicking the brain nervous system, including neuronal dynamics and synaptic plasticity, and is widely used in both supervised and unsupervised learning schemes. ${ }^{29,31,33-36}$ If there are more than two hidden layers constituting ANN, it is specifically called deep neural network (DNN), and using those models to achieve the learning function is called deep learning. ${ }^{33}$ Deep learning approach has a significant advantage in automatically discovering discriminative features from data and learning them compared to traditional machine learning approach, which requires an additional process to extract the features manually and apply them as inputs. ${ }^{31,33,36,37}$ Thus, recently, it

\section{Medical Data}

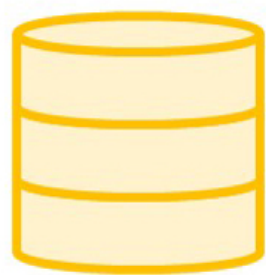

- Biomedical images

- Electrophysiological signals

- Genomic data

\section{Computational Model}

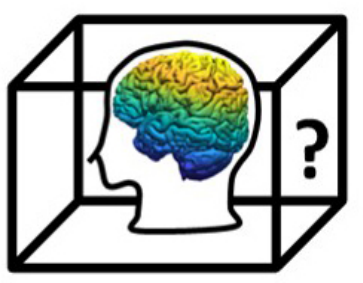

- Machine-learning approach

- Biophysical modeling approach

\section{Personalized Medical Care}

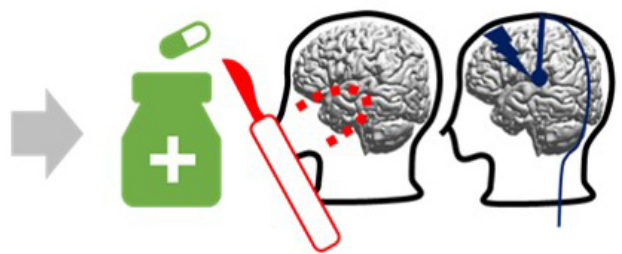

- Accurate diagnosis

- Prognosis prediction

- Optimal treatment

Figure 1. Overview of computational approaches for personalized medicine. Using accumulated large amount of medical data and advanced in-silico techniques, the personalized medical platform could be built to provide accurate diagnosis, prognosis prediction and treatment optimization for individual epilepsy patients. Currently, these computational studies are mainly divided into two approaches: a machine learning approach that implements a model capable of performing a specific function, such as automatic diagnosis, and a biophysical modeling approach that reproduces and simulates the patient's brain network dynamics system itself. 
has been aggressively used in various applications even if it needs higher computational power. For the implementation of deep learning in addition to general DNN model (i.e., multi-layer perceptron), various transformed models, such as convolutional neural network (CNN) specialized for analysis of image data and recurrent neural network (RNN) specialized for analysis of time-series data, have been actively employed. ${ }^{36,37}$ In this section, we briefly review some of the notable studies that have applied machine learning techniques to clinical application of epilepsy.

\section{Classification of seizure and epilepsy types}

Determining seizure types and epilepsy types of individual patients is the first step that should be performed to provide adequate treatment for each patient. Typically, these diagnostic procedures have been conducted by clinicians reviewing multiple forms of data obtained from each patient, including symptoms, etiologies, neuroimages, neurophysiological data such as electroencephalogram (EEG), and etc. Since not only are these tasks time-consuming and laborious, but also recent studies have indicated that it can often be difficult to differentiate the epilepsy types even by experienced clinicians, $^{38}$ the automated models based on the standardized protocols become more and more important.

Recent studies have proposed the machine learning approaches that automatically execute those diagnostic tasks, especially classification of seizure types, and have evaluated their performance. ${ }^{39-45}$ Mainly, using scalp-EEG recordings labeled with seizure types (recorded from multiple patients), some studies have shown that the computational model that trained the spatiotemporal features of the specific seizure classes were able to classify the seizure types with a quite high accuracy. ${ }^{39-43}$ For the implementation of these models in addition to classic machine learning algorithms such as SVM and k-NN, ${ }^{39-40}$ deep learning algorithms such as $\mathrm{CNN}^{41-43}$ have been applied. Notably, Liu and colleagues ${ }^{41}$ have proposed a hybrid bilinear model that combines CNN and RNN. In their hybrid model, CNN and RNN extracted the spatial and temporal features of seizures recorded in scalp EEG, respectively, especially from short-time Fourier transform results of segments with a 1 second time window. Then, these two types of features were combined into second-order statistics through bilinear pooling. The proposed model has classified the seizure types effectively, with F1-scores of $97.4 \%$ and $97.2 \%$ in two datasets, containing 8 and 4 seizure classes, respectively.

Some other studies have built the computational models that cate- gorize seizure types or epilepsy types by training using text-based data containing patients' symptoms. ${ }^{44,45}$ Kassahun and colleagues ${ }^{45}$ have proposed the models that classifies two epilepsy types, temporal lobe epilepsy and extra-temporal lobe epilepsy, based on the ictal symptoms of each patient, using employing two machine learning methods, ontology-based and genetics-based algorithms, and the models have achieved $77.8 \%$ accuracy.

These machine learning-based classification systems can be used to quickly determine disease characteristics of individual patients in a standardized manner, and they can be further applied to suggest drug medications that are appropriate to each patient based on accumulated clinical evidences.

\section{Localization of seizure onset zones (SOZ)}

Investigating SOZ and propagation zones (PZ) is crucial to make accurate diagnosis and treatment plan for each epilepsy patient. Especially when surgical intervention is considered, the localization of $\mathrm{SOZ}$ is essential to determine the surgical resection margin, from which the area that can prevent occurrence of seizures by its removal and does not cause critical impairment to the normal brain functions (i.e., outside of eloquent areas) should be derived. Localization of the SOZ has been mainly conducted by measuring electrophysiological signals containing spontaneous seizures in an invasive manner, such as intracranial EEG (iEEG) or stereotactic EEG (sEEG), and then analyzing the recorded EEG signals.

Recently, several studies have presented the machine learning-based methodology to identify SOZ. ${ }^{46-53}$ In particular, using iEEG recordings from patients, Elahian and colleagues ${ }^{47}$ have built a model to classify each electrode position into $\mathrm{SOZ}$ and non-SOZ. They considered the electrode positions within the resected area and outside the resected area in the patients who showed seizure-free outcome as SOZ and non-SOZ, respectively, and trained the model using the signal characteristics recorded at the corresponding electrodes of two classes. For the training, they extracted certain features from the phase locking values (PLVS) of the signals for each channel, especially PLV between the phase of amplitude of high gamma activity and phase of lower frequency rhythms, and applied them to the logistic regression algorithm. They have demonstrated it in patients with poor postoperative outcomes, some of the SOZ electrodes predicted by the model remained outside the resected area, and the number of non-resected SOZ electrodes correlated with surgical outcomes.

In contrast to the studies that localize SOZ using the recording da- 
ta containing spontaneous seizures ${ }^{47-49}$ as described above, other studies have attempted to perform the same task using only recording data during inter-ictal period. ${ }^{50-53}$ Varatharajah and colleagues ${ }^{51}$ have developed an analytic framework to make localization of SOZ possible, based on multiple biomarkers analyzed from the inter-ictal iEEG recordings, including high frequency oscillation, interictal epi- leptiform discharge, and phase amplitude coupling. The model derived by training the features extracted from the three biomarkers through the SVM algorithm was able to effectively identify the SOZ using 2 hours of interictal recordings (with an average area under ROC curve value of 0.73 , when compared to clinically investigated $\mathrm{SOZ})$.
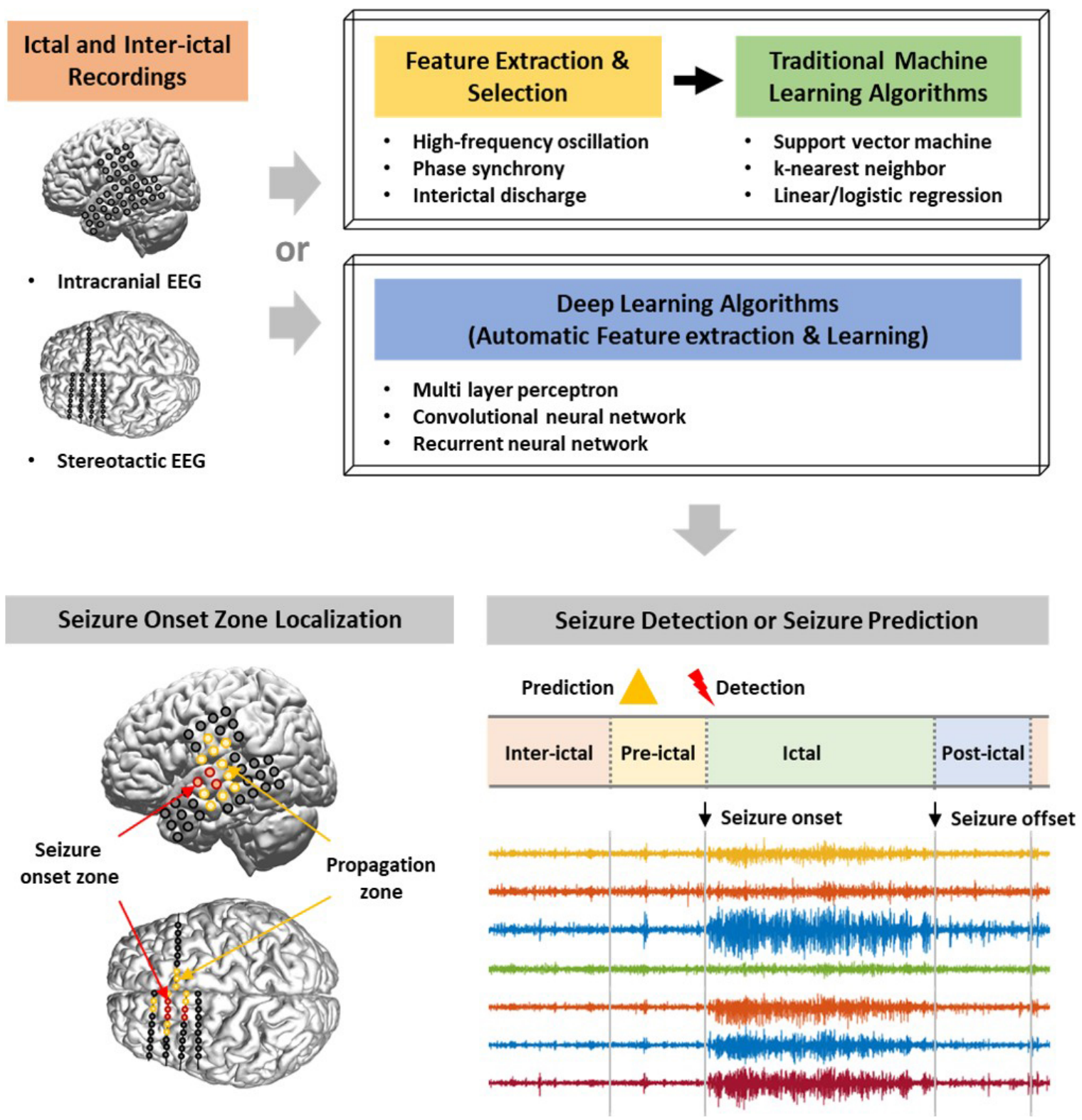

Figure 2. Machine learning-based computational approach. Many recent studies have focused on implementing the computational predictive models to localize SOZs or judge epileptic brain states, such as pre-ictal and ictal onset, by employing traditional machine learning algorithms or deep learning algorithms based on scalp EEG and/or iEEG data recorded from epilepsy patients. Compared to the traditional machine learning approach, which consists of two step processes of manually extracting the features of the data and training the machine by applying the features as inputs, deep learning approach automatically figures out the discriminative features from data and learns them. SOZ, seizure onset zones; EEG, electroencephalogram; iEEG, intracranial EEG. 
With further validation, these machine learning-based SOZ localization systems can be used to assist decision-making process in determining the surgical site when surgical intervention is considered in drug-resistant epilepsy patients (Fig. 2).

\section{Examination of epileptic brain states}

Epileptic brain dynamics can be divided into four states: inter-ictal (period between seizures, i.e., a normal state of patients), pre-ictal (period immediately before the seizure onset), ictal (period during seizure), and post-ictal (period immediately after the seizure). ${ }^{54}$ Investigating the characteristics of each of these states and the transitions between them is significant not only for understanding the pathological mechanisms of the epilepsy, but also treatment and disease management. To date, these studies have been conducted with two main purposes: seizure detection that promptly identifies the seizure onset (time-wise) and seizure prediction that forecast the occurrence of seizure in advance by recognizing the characteristics of the pre-ictal state different from inter-ictal. Recently, numerous studies have proposed computational models that can automatically perform these tasks using accumulated data sets and machine learning algorithms.

Regarding the seizure detection task, many studies have shown that the models trained via traditional machine learning algorithms, $^{55-60}$ especially SVM and k-NN, deep learning algorithms ${ }^{61-65}$ using specific features in time and/or frequency domain based on scalp EEG, or iEEG recordings can successfully detect seizures with multiple types. In particular, Emami and colleagues have proposed a CNN-based seizure detection model. ${ }^{61}$ The model learned the EEG (scalp EEG) characteristics in seizure state and non-seizure state automatically, without additional manual feature extraction procedures, through the supervised learning framework, and was able to detect seizure onset at an average positive rate of $74 \%$ when the entire time series EEG was sequentially input by 1 second (100\% for input by 1 minute). They also have demonstrated that performance of seizure detection depends on the similarity of seizure onset pattern between training data and test data, i.e., new data with a different onset pattern that those of trained data could not be detected well. These results indicated that in order to achieve the model that conducts seizure detection with high performance, it is necessary to train using a large amount of data including various seizure patterns. These computational models with the purpose of seizure onset detection can be used to provide basis for on-demand (closed-loop) stimulation therapies or acute drug treatment.

In relation to the seizure prediction task, numerous studies have reported that the computational models that learned time and/or frequency domain features observed in pre-ictal state was able to predict the occurrence of seizures at least several minutes before the onset. These models have been mainly trained through supervised learning methods, and they were based on a variety of algorithms ranging from classic machine learning algorithms such as $\mathrm{SVM}_{1}^{66-71}$ k-NN, ${ }^{71-73}$ hidden Markov model, ${ }^{74}$ and etc., to deep learning algorithms such as CNN, ${ }^{75-78}$ Long Short-Term Memory ${ }^{70,79}$ (LSTM, a kind of RNN) and their hybrid model, ${ }^{80-82}$ learning the characteristics of the pre-ictal state distinct from inter-ictal. The developed models have shown sensitivity of $80-90 \%$, but it should be noted that each study had a different prediction time (from 5 minutes before to 1 hour before the onset). Currently, studies of seizure prediction have been conducted primarily in the direction of developing patient-specific models based on data obtained from individual patients, rather than developing a generalized model based on lots of data from multiple patients. Given that the signal patterns of iEEG or scalp EEG vary for each patient, this individualized approach may be more useful for clinical applications. Recent studies have implemented seizure prediction models mainly by employing deep learning algorithms. ${ }^{75-82}$ Notably, Daoud and Bayoumi ${ }^{80}$ have developed a model that predicts seizures 1 hour before the onset, with a high accuracy of $99.6 \%$, using long-term scalp EEG. The proposed model employed both CNN and RNN (especially bidirectional LSTM) to learn the spatial and temporal features respectively from raw EEG data and introduced a semi-supervised learning approach based on the transfer learning technique to reduce training time, showing the potential for real-time usage. Meanwhile, Cook and colleagues have demonstrated the feasibility of the implanted seizure advisory system in drug-resistant patients, ${ }^{83}$ in which they implemented a machine learning-based patient-specific algorithm by using iEEG signals recorded for at least 1 month (containing at least 5 leading seizures) at each patient and predicted seizure likelihood based on that algorithm, achieving sensitivities ranging from $65 \%$ to $100 \%$ for each patient. Moreover, Kiral-Kornek and colleagues ${ }^{84}$ have demonstrated feasibility of real-time using wearable devices with low power consumption and long-term reliability by implementing the patient-specific seizure prediction system onto neuromorphic chip. These machine learning-based seizure prediction systems not only help the patients to avoid dangerous situations by alerting them of the likelihood of seizures, but also establish the basis of offering personalized treatment 
by providing each patient's information including frequency, duration, and patterns of seizures to medical institutions (Fig. 2).

\section{Biophysical modeling-based approaches}

As another branch of the computational approaches that is distinct from machine learning-based approach, which create the computational machine to performing specific functions (especially, such as classification or clustering) inspired by the working mechanisms of the neural network, computational modeling approach that reproduces the neural network dynamics per se is also actively underway. ${ }^{85-88}$ These neural network modeling studies are mainly performed in two types of approaches: bottom-up approach of constructing a network based on microscale units including single neurons, synapses and ion channels, and top-down approach of building a whole-brain network model based on macroscale brain connectome to understand its functions and mechanisms. In particular, since a framework to construct the subject-specific brain network model based on individual neuroimaging data has been developed, ${ }^{89-90}$ brain modeling studies of the top-down approach to investigate brain functions and dysfunctions in a personalized manner have been rapidly progressing. In these models at whole brain level, neural mass model, which is a mathematical model that describes the activities of neuronal population rather than the activities of single neurons, is mainly used. These neural mass models are located in each brain region (or, in each sensor location), generating local dynamics, and interact each other by being connected via brain connectivity acquired from the individual brain imaging data. The parameters of each neural mass model that determine the local regional properties can be set through the analysis of individual functional imaging data or through the clinical findings. This personalized modeling approach can be applied to specific diseases, and the developed model could reproduce the pathological characteristics of each patient, such as structural and/or functional alteration of the brain, ${ }^{17,91-93}$ and predict the effects of various therapeutic interventions via systematic simulations in a patient-specific environment. In this section, we briefly review the personalized modeling studies toward clinical application of epilepsy.

\section{Patient-specific whole-brain models}

To date, studies of personalized brain network modeling have been primarily aimed at optimizing surgical intervention strategies by employing retrospective approach, in which the actual surgical site of each patient is compared with the target site derived from the simulations using the model, and those comparison results are analyzed with the surgical outcomes. These patient-specific models have been constructed in two main ways: sensor-based and region-based. In the sensor-based models, neural mass models are positioned in each sensor location, i.e., electrode position of iEEG or scalp EEG, and they are coupled by the functional connectivity analyzed from the corresponding data. ${ }^{9496}$ In the region-based models, neural mass models are located in each brain region and they are coupled by structural brain connectivity analyzed from structural brain imaging data, mainly T1-weighted and diffusion-weighted images. ${ }^{97-102}$ The retrospective modeling studies have demonstrated that poor surgical outcomes are frequently observed when target sites identified by simulations are not sufficiently resected during actual surgery, ${ }^{96,99}$ In other

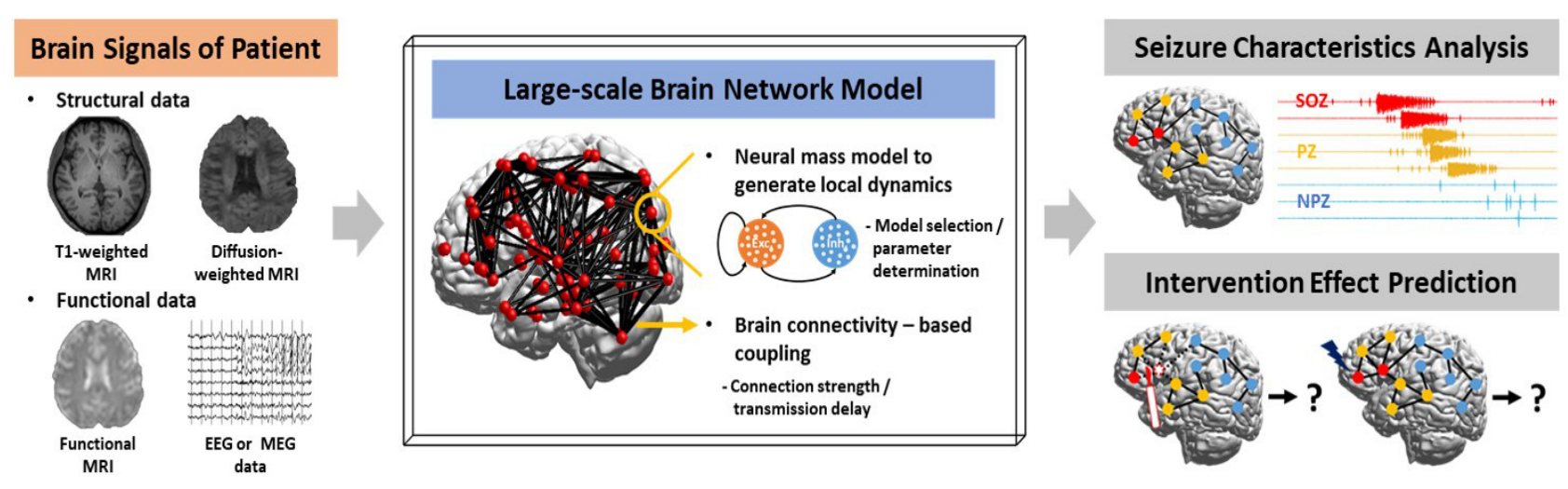

Figure 3. Biophysical modeling-based computational approach. Recent studies based on personalized brain network modeling approach have demonstrated the its feasibility, in which the model can reproduce seizure propagation characteristics of each patient and suggest optimal intervention method for each patient via systematic simulations in the patient-specific environments. 
words, they indicate that in-depth analysis considering network dynamics based on the data is required rather than interpretation of the data itself in order to determine the optimal surgical target.

Jirsa's group has been intensively conducting personalized brain network modeling studies for clinical application, mainly adopting region-based modeling scheme. They have proposed a novel methodology to develop individual brain network models, so called the Virtual Epileptic Patient (VEP) ${ }^{98}$ by incorporating multimodal data from each epilepsy patient, and have also developed and distributed the neuroinformatics platform, The Virtual Brain, to enable the model construction. ${ }^{89-90}$ The VEP model was constructed based on Epileptor, ${ }^{103,104}$ a phenomenological neural mass model replicating seizure characteristics, and patient-specific features including brain connectivity and magnetic resonance imaging lesions, and fit and validated using the individual sEEG recordings. ${ }^{98,99}$ Based on this personalized modeling approach, Jirsa's group has been conducting successive studies in various directions for clinical applications, such as inference of the epileptogenicity of the brain regions, ${ }^{102}$ analysis of the seizure propagation characteristics, ${ }^{99-101}$ and prognostic prediction of the surgical Intervention. ${ }^{100,101}$

Proix et al. ${ }^{99}$ have created the personalized brain network models for 15 epilepsy patients and simulated individual seizure propagation patterns. They also have explained the variability of surgical outcomes using the simulated seizure propagation patterns, demonstrating that the number of regions, which were identified as the $\mathrm{PZ}$ in the simulation but not considered in the pre-surgical evaluation (because they were not investigated by $\mathrm{sEEG}$ ), were correlated with poor surgical outcomes. Some other researchers have performed simulations to derive the surgical target sites that are minimally invasive and effective in suppressing seizures, by applying an in-silico surgical approach to the personalized model, i.e., by investigating the degree of seizure propagation in pre- and post-surgical conditions. ${ }^{100,101}$ An et al. ${ }^{101}$ have proposed a strategy to evaluate the safety of the target sites in order not to cause impairment of normal brain functions, in which they operationalized the safety by the concept of preservation of signal transmission capacity of the brain network, by comparing the simulation-induced response network characteristics in pre- and post-surgical conditions. These research outcomes have shown the possibility that the personalized brain network model could support clinical decision in determining the target site for surgery, and Jirsa's group are currently applying this modeling approach to large-scale clinical trials in order to evaluate its effectiveness.

These personalized network modeling approaches can be applied to investigate the effect of various brain interventions such as neurostimulation in addition to surgical treatment and thus can be utilized to understand the therapeutic mechanisms and suggest optimal treatment method for each patient (Fig. 3).

\section{Conclusions}

In this paper, we have reviewed the computational studies that have been conducted for clinical applications in epilepsy, especially in terms of Al-based approach to generate a computational machine performing specific functions, and biophysical modeling-based approach to replicate neural network dynamics per se. Numerous studies have demonstrated the feasibility of computational approaches that could build a novel medical paradigm with a personalized manner, encompassing diagnosis, prognosis and optimization of treatment, by integrating multimodal data and their analytical results into a single platform. For successful clinical application of the developed computational systems and commercialization through optimization, close interdisciplinary cooperation in various fields including medicine, neuroscience, computer science, and engineering is crucial.

\section{Acknowledgements}

This study was supported by grants from the Basic Science Research Program and Convergent Technology R\&D Program for Human Augmentation through the National Research Foundation of Korea (NRF) funded by the Ministry of Science, Information and Communication Technologies \& Future Planning (2017R1A2A2A050696, 2017R1B5A2086553, 2018M3C1B8016147, 2019M3C1B8090803, and 2020R1A2C2013216) to H. W. Lee. This research was also supported by Basic Science Research Program through the National Research Foundation of Korea (NRF) funded by the Ministry of Education (2020R1I1A1A01073605) to S. An.

\section{Conflict of Interest}

The authors declare that they have no conflicts of interest.

\section{References}

1. Beghi $E$, Giussani $G$, Nichols $E$, et al. Global, regional, and national burden of epilepsy, 1990-2016: a systematic analysis for the Global Burden of Disease Study 2016. Lancet Neurol 2019;18:357-75.

2. Stafstrom CE, Carmant L. Seizures and epilepsy: an overview for neuroscientists. Cold Spring Harb Perspect Med 2015;5:a022426. 
3. Wu Q, Zhao CW, Long Z, Xiao B, Feng L. Anatomy based networks and topology alteration in seizure-related cognitive outcomes. Front Neuroanat 2018;12:25.

4. Allers $\mathrm{K}$, Essue BM, Hackett ML, et al. The economic impact of epilepsy: a systematic review. BMC Neurol 2015;15:245.

5. Jennum P, Gyllenborg J, Kjellberg J. The social and economic consequences of epilepsy: a controlled national study. Epilepsia 2011;52:949-56.

6. Choi HY, Kim SE, Lee HW, Kim EJ. Social behavioral problems and the health-related quality of life in children and adolescents with epilepsy. Psychiatry Investig 2016;13:488-95.

7. Perucca $E$, French J, Bialer M. Development of new antiepileptic drugs: challenges, incentives, and recent advances. Lancet Neurol 2007;6: 793-804.

8. Brodie MJ, Barry SJ, Bamagous GA, Norrie JD, Kwan P. Patterns of treatment response in newly diagnosed epilepsy. Neurology 2012;78:1548-54.

9. Engel J Jr. The current place of epilepsy surgery. Curr Opin Neuro/ 2018; 31:192-7.

10. Jobst $B C$, Cascino $G D$. Resective epilepsy surgery for drug-resistant focal epilepsy: a review. JAMA 2015;313:285-93.

11. Bulacio JC, Jehi $L$, Wong $C$, et al. Long-erm seizure outcome after resective surgery in patients evaluated with intracranial electrodes. Epilepsia 2012;53:1722-30.

12. De Ribaupierre $S$, Delalande 0 . Hemispherotomy and other disconnective techniques. Neurosurg Focus 2008;25:E14.

13. Lee HW, Youngblood MW, Farooque $P$, et al. Seizure localization using three-dimensional surface projections of intracranial EEG power. Neuroimage 2013;83:616-26.

14. Hader WJ, Tellez-Zenteno J, Metcalfe A, et al. Complications of epilepsy surgery: a systematic review of focal surgical resections and invasive EEG monitoring. Epilepsia 2013;54:840-7.

15. Georgiadis I, Kapsalaki EZ, Fountas KN. Temporal lobe resective surgery for medically intractable epilepsy: a review of complications and side effects. Epilepsy Res Treat 2013;2013:752195.

16. Vakharia VN, Duncan JS, Witt JA, Elger CE, Staba R, Engel J Jr. Getting the best outcomes from epilepsy surgery. Ann Neuro/ 2018;83:676-90.

17. Park CH, Choi YS, Kim HJ, et al. Interactive effects of seizure frequency and lateralization on intratemporal effective connectivity in temporal lobe epilepsy. Epilepsia 2018;59:215-25.

18. Boon P, De Cock E, Mertens A, Trinka E. Neurostimulation for drug-resistant epilepsy: a systematic review of clinical evidence for efficacy, safety, contraindications and predictors for response. Curr Opin Neurol 2018;31: 198-210.

19. Yan $H$, Toyota $E$, Anderson $M$, et al. A systematic review of deep brain stimulation for the treatment of drug-resistant epilepsy in childhood. J Neurosurg Pediatr 2018;23:274-84.

20. Geller EB. Responsive neurostimulation: review of clinical trials and insights into focal epilepsy. Epilepsy Behav 2018;88S:11-20.

21. Yang J, Phi JH. The present and future of vagus nerve stimulation. J Korean Neurosurg Soc 2019;62:344-52.
22. Lee HW. Neuromodulation therapy: nonmedical, nonsurgical treatment for intractable epilepsy. In: Holmes MD, ed. Epilepsy Topics. London: IntechOpen, 2014;161-170.

23. Ahn $S$, Jo $S$, Jun $S B$, Lee $H W$, Lee $S$. Study on the mechanisms of seizure-like events suppression effect by electrical stimulation using a microelectrode array. Neuroreport 2017;28:471-8.

24. Ahn S, Jo S, Jun SB, Lee HW, Lee S. Prediction of the seizure suppression effect by electrical stimulation via a computational modeling approach. Front Comput Neurosci 2017;11:39.

25. Niriayo YL, Mamo A, Kassa TD, et al. Treatment outcome and associated factors among patients with epilepsy. Sci Rep 2018;8:1-9.

26. Malmgren $K$, Edelvik $A$. Long-term outcomes of surgical treatment for epilepsy in adults with regard to seizures, antiepileptic drug treatment and employment. Seizure 2017;44:217-24.

27. Anyanwu C, Motamedi GK. Diagnosis and surgical treatment of drug-resistant epilepsy. Brain Sci 2018:8:49.

28. Mohanraj $R$, Brodie MJ. Early predictors of outcome in newly diagnosed epilepsy. Seizure 2013;22:333-44.

29. Awad M, Khanna R. Efficient Learning Machines: Theories, Concepts, and Applications for Engineers and System Designers. New York: Apress, 2015;268.

30. El Morr C, Ali-Hassan H. Analytics in Healthcare: a Practical Introduction. 1st ed. Cham: Springer, 2019.

31. Abbasi B, Goldenholz DM. Machine learning applications in epilepsy. Epilepsia 2019;60:2037-47.

32. Yu KH, Beam AL, Kohane IS. Artificial intelligence in healthcare. Nat Biomed Eng 2018;2:719-31.

33. Valliani AA, Ranti D, Oermann EK. Deep learning and neurology: a systematic review. Neurol Ther 2019;(2):351-65.

34. Suzuki K. Artificial Neural Networks: Methodological Advances and Biomedical Applications. London: IntechOpen, 2011.

35. Al-Shayea QK. Artificial neural networks in medical diagnosis. IJCS/ 2011;8:150-4.

36. Richards BA, Lillicrap TP, Beaudoin $P$, et al. A deep learning framework for neuroscience. Nat Neurosci 2019;22:1761-70.

37. Esteva $A$, Robicquet $A$, Ramsundar $B$, et al. A guide to deep learning in healthcare. Nat Med 2019;25:24-9.

38. Panayiotopoulos CP. The Epilepsies: Seizures, Syndromes and Management. Oxfordshire: Bladon Medical Publishing, 2005;1-26.

39. Saputro IRD, Maryati ND, Solihati SR, Wijayanto I, Hadiyoso S, Patmasari R. Seizure type classification on EEG signal using support vector machine. J Phys: Conf Ser 2019;1201:012065.

40. Roy S, Asif U, Tang J, Harrer S. Machine learning for seizure type classification: setting the benchmark [Internet]. Southbank: IBM Research, [cited 2020 Jun 10]. Available at: https://arxiv.org/abs/1902.01012.

41. Liu T, Truong ND, Nikpour A, Zhou L, Kavehei O. Epileptic seizure classification with symmetric and hybrid bilinear models. IEEE J Biomed Health Inform 2020 Apr 2. doi: 10.1109/JBHI.2020.2984128.

42. Raghu, Sriraam N, Temel Y, Rao SV, Kubben PL. A convolutional neural 
network based framework for classification of seizure types. Conf Proc IEEE Eng Med Biol Soc 2019;2019:2547-50.

43. Ahmedt-Aristizabal D, Nguyen K, Denman S, Sridharan S, Dionisio S, Fookes C. Deep motion analysis for epileptic seizure classification. Conf Proc IEEE Eng Med Biol Soc 2018;2018:3578-81.

44. Pereira L, Rijo R, Silva C, Agostinho M. ICD9-based text mining approach to children epilepsy classification. Procedia Technology 2013;9:1351-60.

45. Kassahun $Y$, Perrone R, De Momi $E$, et al. Automatic classification of epilepsy types using ontology-based and genetics-based machine learning. Artif Intell Med 2014;61:79-88.

46. Si Y. Machine learning applications for electroencephalograph signals in epilepsy: a quick review. Acta Epileptologica 2020;2:1-7.

47. Elahian B, Yeasin M, Mudigoudar B, Wheless JW, Babajani-Feremi A. Identifying seizure onset zone from electrocorticographic recordings: a machine learning approach based on phase locking value. Seizure 2017;51:35-42.

48. Grinenko O, Li J, Mosher JC, et al. A fingerprint of the epileptogenic zone in human epilepsies. Brain 2018;141:117-31.

49. Murin Y, Kim J, Parvizi J, Goldsmith A. SozRank: a new approach for localizing the epileptic seizure onset zone. PLoS Comput Biol 2018; 14:e1005953.

50. Akter MS, Islam MR, limura Y, et al. Multiband entropy-based feature-extraction method for automatic identification of epileptic focus based on high-frequency components in interictal iEEG. Sci Rep 2020;10:1-17.

51. Varatharajah $Y$, Berry $B$, Cimbalnik J, et al. Integrating artificial intelligence with real-time intracranial EEG monitoring to automate interictal identification of seizure onset zones in focal epilepsy. J Neural Eng 2018;15: 046035.

52. Nissen IA, Stam CJ, van Straaten ECW, et al. Localization of the epileptogenic zone using interictal MEG and machine learning in a large cohort of drug-resistant epilepsy patients. Front Neurol 2018;9:647.

53. Baud MO, Kleen JK, Anumanchipalli GK, et al. Unsupervised learning of spatiotemporal interictal discharges in focal epilepsy. Neurosurgery 2018;83:683-91.

54. Kitano LAS, Sousa MAA, Santos SD, Pires R, Thome-Souza S, Campo AB. Epileptic Seizure Prediction from EEG Signals Using Unsupervised Learning and a Polling-Based Decision Process. In: Kưrková $V_{\text {, }}$ Manolopoulos Y, Hammer B, lliadis L, Maglogiannis I, eds. Artificial Neural Networks and Machine Learning - ICANN 2018. Cham: Springer, 2018; 117-126.

55. Fergus $P$, Hussain A, Hignett D, Al-Jumeily D, Abdel-Aziz K, Hamdan H. A machine learning system for automated whole-brain seizure detection. Applied Computing and Informatics 2016;12:70-89.

56. Kang Lövgren S, Rosquist C. Machine learning methods for EEG-based epileptic seizure detection [dissertation]. KTH Loyal Institute of Technology; 2019. 1-38.

57. Chan AM, Sun FT, Boto EH, Wingeier BM. Automated seizure onset detection for accurate onset time determination in intracranial EEG. Clin Neurophysiol 2008;119:2687-96.
58. Rezaee Kh, Azizi E, Haddadnia J. Optimized seizure detection algorithm: a fast approach for onset of epileptic in EEG signals using GT discriminant analysis and K-NN classifier. J Biomed Phys Eng 2016;6:81-94.

59. Wang $L$, Xue W, Li $Y$, et al. Automatic epileptic seizure detection in EEG signals using multi-domain feature extraction and nonlinear analysis. Entropy 2017;19:222.

60. Lee J, Park J, Yang S, et al. Early seizure detection by applying frequency-based algorithm derived from the principal component analysis. Front Neuroinform 2017;11:52.

61. Emami A, Kunii N, Matsuo T, Shinozaki T, Kawai K, Takahashi H. Seizure detection by convolutional neural network-based analysis of scalp electroencephalography plot images. Neuroimage Clin 2019;22:101684.

62. Zhou M, Tian C, Cao R, et al. Epileptic seizure detection based on EEG signals and CNN. Front Neuroinform 2018;12:95.

63. Hussein R, Palangi $H$, Ward RK, Wang Z. Optimized deep neural network architecture for robust detection of epileptic seizures using EEG signals. Clin Neurophysiol 2019;130:25-37.

64. Cho KO, Jang HJ. Comparison of different input modalities and network structures for deep learning-based seizure detection. Sci Rep 2020;10:122.

65. Jang HJ, Cho KO. Dual deep neural network-based classifiers to detect experimental seizures. Korean J Physiol Pharmacol 2019;23:131-9.

66. Williamson JR, Bliss DW, Browne DW, Narayanan JT. Seizure prediction using EEG spatiotemporal correlation structure. Epilepsy Behav 2012; 25:230-8.

67. Rasekhi J, Mollaei MR, Bandarabadi M, Teixeira CA, Dourado A. Preprocessing effects of 22 linear univariate features on the performance of seizure prediction methods. J Neurosci Methods 2013;217:9-16.

68. Park Y, Luo L, Parhi KK, Netoff T. Seizure prediction with spectral power of EEG using cost-sensitive support vector machines. Epilepsia 2011; 52:1761-70.

69. Zhang Z, Parhi KK. Low-complexity seizure prediction from iEEG/sEEG using spectral power and ratios of spectral power. IEEE Trans Biomed Circuits Syst 2016;10:693-706.

70. Dadgar-Kiani E, Alkan C, Shameli A. Applying machine learning for human seizure prediction. Stanford: Stanford University; 2016.

71. Usman SM, Hassan A. Efficient prediction and classification of epileptic seizures using EEG data based on univariate linear features. $J C P$ 2018;13:616-21.

72. Ibrahim SW, Djemal R, Alsuwailem A, Gannouni S. Electroencephalography (EEG)-based epileptic seizure prediction using entropy and K-nearest neighbor (KNN). Communications in Science and Technology 2017;2;6-10.

73. Wang S, Chaovalitwongse WA, Wong $S$. Online seizure prediction using an adaptive learning approach. IEEE Trans Knowl Data Eng 2013; 25:2854-66.

74. Wong $S$, Gardner AB, Krieger AM, Litt B. A stochastic framework for evaluating seizure prediction algorithms using hidden Markov models. J Neurophysiol 2007;97:2525-32.

75. Hussein R, Ahmed MO, Ward R, Wang ZJ, Kuhlmann L, Guo Y. Human intracranial EEG quantitative analysis and automatic feature learning for 
epileptic seizure prediction [Internet]. Vancouver: University of British Columbia, [cited 2020 Jun 10]. Available at: https://arxiv.org/abs/1904.03603.

76. Truong ND, Zhou L, Kavehei O. Semi-supervised seizure prediction with generative adversarial networks. Conf Proc IEEE Eng Med Biol Soc 2019; 2019:2369-72.

77. Truong ND, Nguyen AD, Kuhlmann $L$, et al. Convolutional neural networks for seizure prediction using intracranial and scalp electroencephalogram. Neural Netw 2018;105:104-11.

78. Khan $H$, Marcuse $L$, Fields $M$, Swann $K$, Yener B. Focal onset seizure prediction using convolutional networks. IEEE Trans Biomed Eng 2017; 65:2109-18.

79. Tsiouris KM, Pezoulas VC, Zervakis $M$, Konitsiotis S, Koutsouris DD, Fotiadis DI. A long short-term memory deep learning network for the prediction of epileptic seizures using EEG signals. Comput Biol Med 2018;99:24-37.

80. Daoud H, Bayoumi MA. Efficient epileptic seizure prediction based on deep learning. IEEE Trans Biomed Circuits Syst 2019;13:804-13.

81. Shahbazi $M$, Aghajan H. A generalizable model for seizure prediction based on deep learning using CNN-LSTM architecture. 2018 IEEE Global Conference on Signal and Information Processing (GlobalSIP). Anaheim (CA): USA. 2018. p. 469-473.

82. Thodoroff $P$, Pineau J, Lim A. Learning robust features using deep learning for automatic seizure detection. Proceedings of Machine Learning and Healthcare 2016. Children's Hospital LA, USA. Cambridge: PMLR, 2016;178-190.

83. Cook MJ, O'Brien TJ, Berkovic SF, et al. Prediction of seizure likelihood with a long-term, implanted seizure advisory system in patients with drug-resistant epilepsy: a first-in-man study. Lancet Neurol 2013;12: 563-71.

84. Kiral-Kornek I, Roy S, Nurse E, et al. Epileptic seizure prediction using big data and deep learning: toward a mobile system. EBioMedicine 2018; 27:103-11.

85. Holt $A B$, Netoff $T I$. Computational modeling of epilepsy for an experimental neurologist. Exp Neurol 2013;244:75-86.

86. Lytton WW. Computer modelling of epilepsy. Nat Rev Neurosci 2008;9: 626-37.

87. Wendling F, Benquet $P$, Bartolomei $F$, Jirsa V. Computational models of epileptiform activity. J Neurosci Methods 2016;260:233-51.

88. Ahn S, Jun SB, Lee HW, Lee S. Computational modeling of epileptiform activities in medial temporal lobe epilepsy combined with in vitro experiments. J Comput Neurosci 2016;41:207-23.

89. Sanz Leon P, Knock SA, Woodman MM, et al. The Virtual Brain: a simulator of primate brain network dynamics. Front Neuroinform 2013;7:10.

90. Sanz-Leon P, Knock SA, Spiegler A, Jirsa VK. Mathematical framework for large-scale brain network modeling in The Virtual Brain. Neuroimage 2015;111:385-430.

91. Kim HJ, Lee JH, Park CH, et al. Role of language-related functional connectivity in patients with benign childhood epilepsy with centrotemporal spikes. J Clin Neurol 2018;14:48-57.

92. Kim H, Choi Y, Joung HY, et al. Structural and functional alterations at pre-epileptic stage are closely associated with epileptogenesis in pilocarpine-induced epilepsy model. Exp Neurobiol 2017;26:287-94.

93. Kim SE, Lee JH, Chung HK, Lim SM, Lee HW. Alterations in white matter microstructures and cognitive dysfunctions in benign childhood epilepsy with centrotemporal spikes. Eur J Neurol 2014;21:708-17.

94. Woldman W, Schmidt $H$, Abela $E$, et al. Dynamic network properties of the interictal brain determine whether seizures appear focal or generalised. Sci Rep 2020;10:7043.

95. Goodfellow M, Rummel C, Abela E, Richardson MP, Schindler K, Terry JR. Estimation of brain network ictogenicity predicts outcome from epilepsy surgery. Sci Rep 2016;6:29215.

96. Sinha N, Dauwels J, Kaiser M, et al. Predicting neurosurgical outcomes in focal epilepsy patients using computational modelling. Brain 2017;140: 319-32.

97. Hutchings F, Han CE, Keller SS, Weber B, Taylor PN, Kaiser M. Predicting surgery targets in temporal lobe epilepsy through structural connectome based simulations. PLOS Comput Biol 2015;11:e1004642.

98. Jirsa VK, Proix T, Perdikis D, et al. The virtual epileptic patient: individualized whole-brain models of epilepsy spread. Neuroimage 2017;145(Pt B):377-88.

99. Proix T, Bartolomei F, Guye M, Jirsa VK. Individual brain structure and modelling predict seizure propagation. Brain 2017;140:641-54.

100. Olmi S, Petkoski S, Guye M, Bartolomei F, Jirsa V. Controlling seizure propagation in large-scale brain networks. PLoS Comput Biol 2019; 15:e1006805.

101. An S, Bartolomei F, Guye M, Jirsa V. Optimization of surgical intervention outside the epileptogenic zone in the Virtual Epileptic Patient (VEP). PLOS Comput Biol 2019;15:e1007051.

102. Hashemi M, Vattikonda AN, Sip V, et al. The Bayesian Virtual Epileptic Patient: a probabilistic framework designed to infer the spatial map of epileptogenicity in a personalized large-scale brain model of epilepsy spread. Neuroimage 2020;217:116839.

103. Jirsa VK, Stacey WC, Quilichini PP, Ivanov Al, Bernard C. On the nature of seizure dynamics. Brain 2014;137(Pt 8):2210-30.

104. Proix T, Bartolomei F, Chauvel P, Bernard C, Jirsa VK. Permittivity coupling across brain regions determines seizure recruitment in partial epilepsy. J Neurosci 2014;34:15009-21. 